\title{
La ciencia política de fin de siglo
}

"La tarea más urgente y difícil que espera hoy a la ciencia política es analizar y eventualmente cuestionar la propia ideología de la política científica, examinando su significado histórico y actual, destacando sus límites y condiciones de practicabilidad, indicando sus posibles líneas de desarrollo."

Norberto Bobbio1

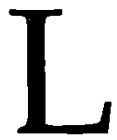

a interrogante que nos proponemos dilucidar en esta introducción se refiere al efecto que las recientes transformaciones a escala mundial, producto sobre todo de la distensión del bloque comunista de Europa del Este, puede tener en el desarrollo inmediato y futuro de la ciencia política.

En el lapso de apenas unos cuantos años el género humano ha visto transformaciones en la escena mundial que, en otros tiempos y circunstancias, hubieran implicado ciclos de varias décadas para desarrollarse. El colapso del viejo sistema soviético, el fin de la guerra fría, la revaloración de la democracia y del mercado, la multiplicación de los centros hegemónicos y la reestructuración de la economía-mundo, son tan sólo los cambios visibles de un complejo proceso que escapa a cualquier posibilidad de comprensión global.

Por el momento sólo es posible determinar algunos efectos producto de dichas transformaciones. Así, por ejemplo, el colapso del comunismo replantea no sólo el papel del mundo soviético, sino también los propios presupuestos de la defensa militar y la confrontación entre Estados Unidos y la Unión Soviética. El fin de la guerra fría se expresa sobre todo en una disminución de los conflictos regionales (Afganistán, Angola, 
Centroamérica, etcétera) producto de la rivalidad de los superpoderes. La Unión Soviética, como polo hegemónico, cede su lugar a Europa Occidental (en particular Alemania) y a Asia del Este (en particular Japón), con lo que la rivalidad militar se desplaza cada vez más hacia la competencia económica y el intercambio comercial.2

En términos económicos, el triunfo de la economía de mercado, por una parte, y de las políticas neoliberales, por la otra, configuran un mundo de intercambios y alianzas económicas entre los centros de poder en el cual no parecen tener mayores beneficios los países del Tercer Mundo. En relación con los países de Europa Oriental, que obviamente capturarán los recursos económicos de la Comunidad Europea en el futuro inmediato, los países de América Latina, África y Asia, salvo excepciones, verán agravarse sus problemas de integración económica y desarrollo interno, pues su papel de proveedores de materias primas y de mano de obra barata les confiere un lugar claramente subordinado y dependiente en una economía mundial cada vez más automatizada. ${ }^{3}$

Considerando la magnitud de estos cambios, resulta lógico esperar que su efecto se dejará sentir en mayor o menor medida en todas las esferas del quehacer humano y en todos los subsistemas sociales. Es precisamente en este contexto donde nuestra interrogante inicial cobra sentido. Siendo las ciencias sociales las disciplinas científicas que supuestamente proporcionan la interpretación más objetiva de las implicaciones de estos cambios, resulta significativo preguntarse en qué medida se verán afectadas en su patrón evolutivo como consecuencia, ya sea de las nuevas condiciones mundiales o de la exigencia de dar cuenta de los muchos fenómenos inéditos que experimentamos en la actualidad.

Más específicamente, nos interesa reflexionar en estas páginas sobre el desarrollo posible de la ciencia política, cuya rápida evolución desde los años cincuenta hasta la fecha la convierte en una de las ciencias sociales más importantes. Suponemos de entrada que el cambiante contexto mundial afectará sensiblemente no sólo a los contenidos y paradigmas dominantes hasta ahora dentro de la disciplina, sino también a los patrones de profesionalización e institucionalización que la han caracterizado. Obviamente, dicho efecto sólo puede establecerse diferencialmente por cuanto la disciplina muestra estadios de desarrollo muy diversos de un país a otro o de una región geográfica a otra. Asimismo, en un análisis de este tipo, es necesario ser cuidadosos en evitar adjudicar todos los cambios en la disciplina al contexto de referencia, por cuanto la evolución de los paradigmas dentro de la ciencia política tiene que ver también con ciertas reglas implícitas a toda disciplina científica que, más que referirse a factores coyunturales, tiene relación con la propia acumula- 
ción de los saberes y la depuración de sus referentes teóricos.

\section{II}

Para proceder con este análisis consideraremos dos aspectos centrales: 1) las diferentes etapas evolutivas de la ciencia política y 2) las diferentes áreas definitorias de la ciencia política.

Respecto al primer punto, podemos establecer diferentes etapas de desarrollo de la ciencia política a partir de cuando menos dos aspectos: $a$ ) el nivel de autonomía de la ciencia política respecto a otras disciplinas, y b) el grado de institucionalización de la disciplina.

El nivel de autonomía se refiere sobre todo a si la reflexión de la realidad política ha alcanzado o no un status científico; es decir, si la ciencia política se ha convertido en el "monopolio" del discurso especializado (científico) sobre lo político, siendo reconocida como autónoma respecto a otras disciplinas (sociología, filosofía, historia, etcétera). Por status científico entiendo simplemente el estudio o investigación de los diferentes aspectos de la realidad política con las metodologías propias de las ciencias empíricas. 4 Las diferentes etapas de la ciencia política pueden ser establecidas así por la mayor o menor autonomía alcanzada por la disciplina.

En lo que respecta a la institucionalización de la disciplina, nos referimos simplemente a si la ciencia política ha alcanzado un lugar en la vida académica del país, lo cual se determina por la existencia o no de publicaciones especializadas, licenciaturas y posgrados, institutos de investigación, etcétera. 5

Considerando el nivel en que se presentan estos dos aspectos podemos establecer cuando menos cuatro etapas evolutivas de la disciplina: 1) precientífica, 2) baja institucionalización, 3) alta institucionalización pero en busca de su autonomía, y 4) consolidada.

Ahora bien, si deseamos determinar el efecto de las transformaciones mundiales actuales en el desarrollo de la ciencia política, debemos establecer previamente para cada contexto nacional o área geográfica el estadio en el que se encuentra la disciplina (véase el esquema 1). De acuerdo con una revisión muy somera de la literatura existente sobre el tema, podríamos establecer la siguiente clasificación tentativa: 6

a) La ciencia política empírica conoce sus mejores desarrollos en Estados Unidos, Canadá y en algunos países de Europa Occidental, tales como Italia, Inglaterra, Alemania, Francia y, de manera más reciente, España. En todos estos casos la ciencia política ha logrado plena autonomía 
y en conjunto concentran alrededor del $90 \%$ de la producción mundial en la disciplina.

b) En un estadio inferior deben colocarse el resto de los países de Europa Occidental, varios países de América Latina (México, Brasil, Argentina, Chile, Uruguay), así como los casos de Israel, Japón, India y Australia. En todos estos países la ciencia política ha conocido importantes desarrollos; el nivel de institucionalización es elevado y existen aportaciones originales a la disciplina. En contrapartida, no puede afirmarse que la ciencia política haya alcanzado aquí plena autonomía, pues aún se debaten su cientificidad y especificidad.

c) Los países donde la ciencia política empírica ha conocido algún nivel de profesionalización, pero carece casi por completo de status científico son sobre todo los de Europa del Este, el resto de los países de América Latina, buena parte del Medio Oriente y de Asia.

d) Finalmente, la ciencia política se encuentra en una fase precientífica o simplemente no existe en prácticamente todo el continente africano y en algunos países de Asia.

Pero si nos interesa ver las variaciones específicas en el desarrollo de la disciplina para cada contexto, es necesario distinguir el segundo aspecto sugerido antes: las diferentes áreas definitorias de la ciencia política.

\section{III}

Para fines de la exposición sugiero considerar las siguientes tres áreas definitorias de la ciencia política: a) contenidos y temáticas, b) paradigmas dominantes y c) concepción de la ciencia política.

Nuestra hipótesis explicativa sobre este punto puede plantearse en los siguientes términos: el efecto de las transformaciones mundiales actuales en el desarrollo de la ciencia política será mayor, cuanto mayores sean las variaciones que presenten cada una de estas áreas en cada contexto nacional.

Para comenzar con la primera de las áreas señaladas, es decir, los contenidos y temáticas de la ciencia política, es posible advertir grandes transformaciones a escala mundial, incluyendo a Europa del Este. Recientemente, Carole Pateman, actual presidente de la Asociación Internacional de Ciencia Política, ha exhortado a la comunidad politóloga a "construir una nueva ciencia política para un nuevo mundo". Entre el elenco de las nuevas temáticas que ya ocupan el interés de un creciente número de politólogos, Pateman señala las siguientes: las transformaciones y con- 
secuencias de los sistemas políticos; las condiciones para la democracia y el desempeño democrático (nuevos cleavages sociales, problemas de la modernización); la política económica y su impacto social; la integración económica, el nuevo orden económico y el papel del Estado; las posibilidades de un Estado global; las transiciones poscomunistas; etcétera.7

De acuerdo con lo anterior, la ciencia política muestra en la actualidad un renovado interés por la política comparada y la macropolítica. Ello es importante ya que la tendencia dominante en la ciencia política empírica la había llevado, en virtud del rigor científico y el culto al dato, a los terrenos de la especialización y parcialización extremas, por no hablar de la creciente trivialidad o irrelevancia de muchos de sus temas. 8 Pero de ser así, la ciencia política deberá volver a discutir sus presupuestos de base y definir sus propias fronteras respecto a la teoría política, la filosofía política y otras ciencias sociales. De ello nos ocuparemos más adelante.

Pero quizá más importante que este primer aspecto es identificar cómo las actuales transformaciones mundiales afectan a los paradigmas dominantes de la ciencia política en cada contexto nacional.

Sin embargo, para evitar confusiones, conviene advertir previamente algunas características propias de los paradigmas dominantes en las ciencias sociales. Siguiendo a Thomas S. Kuhn, quien ha desarrollado el análisis más sólido sobre este tema, un paradigma define una etapa o un estadio de una ciencia. En ese sentido, el paradigma constituye un conjunto particular de ideas filosóficas, teorías científicas y normas metodológicas que predominan en un estadio de una ciencia o de varias ciencias y que lo distingue de otros. 9

Sin embargo, esta interpretación presenta algunas dificultades cuando lo que se considera son las ciencias sociales. En el modelo de "ciencia normal" de Kuhn (período en el que los académicos están intensamente ocupados en la tarea de la "articulación paradigmática"), la acumulación de datos para apoyar el paradigma dominante convencionalmente aceptado, es tan engañosa como su imagen contraria de "revolución científica". En efecto, las ciencias sociales no están dominadas por un sólo paradigma, y nuevas teorías modifican -si no es que sustituyen por completo- las viejas. Para el caso de las ciencias sociales, la "ciencia normal" no es necesariamente estática en lo teórico, como lo implica el modelo de Kuhn, sino que con frecuencia está caracterizada por reducciones incrementales en los residuos inexplicables y por refinamientos en las teorías existentes. 10

En ese sentido, los avances o cambios recientes en el análisis de los 
aspectos políticos no necesariamente tienen que ver con lo que Kuhn define como "revolución científica", sino que con frecuencia constituyen avances mucho más modestos en la comprensión de estos fenómenos que buscamos entender. Tienen que ver más con este último interés que con la búsqueda explícita de nuevas y fuertes rupturas teóricas.

Considerando este aspecto según los diferentes contextos nacionales, podemos observar diferencias interesantes. Así, por ejemplo, mientras que en Estados Unidos y en Europa Occidental, es decir, allí donde la ciencia política ha logrado consolidarse, no existe un paradigma dominante y parece que la llamada por David Easton "etapa poscomportamentista" (es decir, una etapa dominada por el cuestionamiento de los enfoques tradicionales de la ciencia política empírica, pero también por un amplio pluralismo teórico y metodológico) 11 continuará aún por largo tiempo, en los países de Europa del Este y en otros contextos nacionales (presumiblemente México, Brasil, Argentina y otros países latinoamericanos), donde el marxismo llegó a ser la concepción del mundo dominante entre los científicos sociales al grado de entorpecer el desarrollo de otras perspectivas, se vive en la actualidad un verdadero vuelco ideológico y, en consecuencia, teórico.

En el caso de estos últimos países podemos observar un claro intento por incorporar en la ciencia política local metodologías más empíricas y sofisticadas, elaboradas originalmente en Estados Unidos y en Europa. Así, por ejemplo, en países como México o Brasil existe hoy más lugar que en el pasado, tanto para las metodologías funcionalistas, así como para las perspectivas racionalistas (v.gr. teoría de juegos, elección pública, etcétera), no obstante que las difíciles condiciones económicas de estos países frenan o retardan la evolución de las metodologías y técnicas más refinadas. Con todo, el nuevo interés en estas perspectivas largamente ausentes en estos países así como el menor prejuicio hacia ellas, nos permite predecir que el fin del marxismo como paradigma dominante en la práctica será importante para avanzar en la autonomía de la ciencia política y, en consecuencia, en su consolidación.

En efecto, en virtud de la fuerte influencia del materialismo histórico la ciencia política en los paises en desarrollo, y sobre todo en América Latina, se perdió en la interdisciplinariedad, por lo que cuestiones como el papel de la política, el poder y el Estado fueron reducidas a aspectos secundarios y dependientes de factores socioeconómicos. En el futuro, una de las tareas de la ciencia política en estos países es precisamente generar un entendimiento comparativo sustancial del papel de la política en el desarrollo. 12

En lo que respecta a los países de Europa del Este, en un artículo 
reciente Gabriel Almond documenta la apertura de los rusos hacia los métodos y técnicas originadas por la ciencia política estadunidense y europea. Esta tendencia parece hoy irreversible.13

A ello debe añadirse la existencia de mejores condiciones estructurales para el desarrollo de la investigación politológica en varios de estos países. En efecto, considerando que existe una relación estrecha entre "campo científico" y "campo político", cabe esperar que la democratización gradual de Europa del Este permitirá una reflexión de la política más autónoma o menos comprometida con la élite en el poder y menos mediada por los principios ideológicos del régimen. 14

En el caso de los países que aún no conocen un desarrollo de la ciencia política o donde la reflexión de lo político se encuentra en un estadio precientífico (presumiblemente África y buena parte de Asia y de Oriente Medio), el tipo de problemas es otro. Como señalan Gunnell y Easton, el principal problema aquí parece ser cómo afirmar una ciencia política más vinculada con sus problemas políticos, pues la incorporación de paradigmas externos parece estar divorciada de sus problemas locales; es decir, se realiza un balance crítico de su aplicabilidad y utilidad. En efecto, el imperialismo de las ideas puede dominar tan profundamente la ciencia política local en países donde está menos desarrollada, al grado de hacer que prácticas de investigación teórica y empírica inadecuadas para la sociedad receptora suplanten a las que se estaban formando localmente. 15

Es posible extraer una primera conclusión de lo expuesto hasta aquí: al modificarse estas áreas de la ciencia política como resultado de las transformaciones mundiales actuales, es posible prever también cambios sustanciales en la profesionalización de la disciplina ahí donde todavía muestra desarrollos insuficientes, pero sobre todo en América Latina y en Europa del Este.

Pero quizá el análisis más útil para observar la relación entre las actuales transformaciones y la ciencia política consiste en determinar en cada contexto nacional si la ciencia política ha visto modificaciones en su propia concepción o forma de entenderse.

\section{IV}

Sobre esta última área definitoria de la ciencia política sólo puedo mencionar aquí lo que parece una tendencia dominante, sobre todo en Estados Unidos y en algunos países europeos.

Siguiendo a Easton, la ciencia política en Estados Unidos ha pasado 
en los últimos tiempos por dos momentos de desarrollo que la obligan en la actualidad a redefinir su propia concepción: $a$ ) una fase de crisis del programa original de la disciplina, tal y como se sustentó en la segunda posguerra, y $b$ ) una etapa de crítica posempiricista de la ciencia política. 16

Como es sabido, el programa original de la ciencia política fue delimitado por la corriente comportamentista, con base en los siguientes principios originados en el neopositivismo: a) explicaciones basadas en leyes generales, $b$ ) objetividad y neutralidad valorativa, $c$ ) métodos cuantitativos y estadísticos, $d$ ) sistematicidad y acumulación teórica. Posteriormente, dadas las elevadas expectativas de cientificidad que estos principios implicaban y su impracticabilidad real en el campo de las ciencias sociales, surgieron importantes críticas a la ciencia política tanto desde dentro como desde afuera de la disciplina. Así, por ejemplo, se puso en evidencia que las ciencias sociales tienen una idea de progreso menos clara que la que existe en las ciencias exactas; es decir, muchas de sus teorías no sugieren avance o acumulación teórica. En segundo lugar, estas disciplinas se ven atravesadas por componentes normativos propios de las visiones del mundo que no pueden ser neutralizadas, sino que se infiltran en las tradiciones de investigación, condicionando las propias teorías. Finalmente, en las ciencias sociales nc existen leyes en el sentido fuerte del término; es decir, proposiciones que estipulen relaciones (condicionamientos) invariantes, de validez universal, sino sólo proposiciones que atestiguan regularidades inductivas, generalizaciones empíricas, delimitadas en el tiempo y en el espacio. A ello debe añadirse que la ciencia política no dispone de un cuerpo teórico común y aceptado por todos ni de una concepción única de la explicación científica o de la racionalidad propia de los fenómenos políticos, ni mucho menos de una sola modalidad de control de las teorías. 17

Ciertamente, el debate en torno a la cientificidad de las ciencias sociales está lejos de haberse agotado. En todo caso, lo que nos interesa subrayar aquí es que después de estos dos momentos de desarrollo de la ciencia política, parece ganar cada vez mayor consenso la idea de debilitar o hacer flexibles las fronteras tradicionales de la ciencia política y de la filosofía política, para superar así la llamada "tragedia" de la ciencia política, según una conocida interpretación. 18

Para algunos autores, las diferencias entre la ciencia política y la filosofía política son tan sólo aparentes o de grado: ambas disciplinas tratan de estudiar la realidad política; ambas están involucradas con juicios de valor; ambas trabajan con teorías y evidencias empíricas; etcétera.19 En este orden de ideas, se sostiene que con el debilitamiento de las fronteras 
existentes entre estas dos disciplinas, la ciencia política tradicional y con pretensiones de hard science, empeñada hasta ahora en alcanzar el conocimiento objetivo de la vida política, puede superar su limitada atención a los problemas más glcbales o de macropolítica. Asimismo, al tornarse flexible la idea tradicional de la neutralidad valorativa, la ciencia política puede enfrentar mejor los problemas cruciales de nuestro tiempo, como la crisis de las instituciones democráticas, el papel del Estado en las cuestiones sociales, etcétera.

Una posición menos radical hablaría simplemente de la necesidad de una mayor comunicación entre la ciencia política y la filosofía política. Desde esta perspectiva, la filosofía política, entendida como toda reflexión sobre el fenómeno político que no se limita a estudiar el comportamiento "observable" de los actores políticos y el funcionamiento de los sistemas políticos, sino que también problematiza los medios, fines y sentido de la experiencia política, procuraría a la ciencia política las visiones de la política (o, de manera más general, del hombre, de la sociedad o de la historia) que son el presupuesto de cualquier investigación en ciencias sociales. Por otra parte, la filosofía política permite al politólogo adquirir una mayor conciencia sobre las categorías filosófico-políticas empleadas en su trabajo. Por su parte, la ciencia política ofrece a la filosofía política una ayuda nada desdeñable derivada de lo que las explicaciones causales permiten para la reflexión filosófica. Por otra parte, los conocimientos causales que produce la ciencia política pueden convertirse en el soporte o simplemente en el refuerzo de la plausibilidad de las teorías políticas. Son precisamente las teorías empíricas las que proporcionan los conocimientos causales indispensables a las teorías políticas, mediante las cuales buscamos comprender el sentido de una fase histórica, de una época, y de influir, mediante su circulación, el espíritu público de la sociedad en que vivimos. 20

En una línea cercana a la anterior, Almond ha subrayado recientemente la necesidad de arribar a un lugar de encuentro entre los diferentes sectores y escuelas involucradas en la reflexión de la política, independientemente de su origen más o menos científico, a fin de integrarlas y garantizar la acumulatividad de los saberes producidos. Dicho lugar de encuentro no es otro que la teoría política o, para decirlo con la propia metáfora empleada por Almond, la "cafetería del centro" que abastece a las diferentes "mesas separadas" dentro de la disciplina.21

Esta pretensión, sin embargo, parece francamente lejana de la realidad. En la práctica, los sectores más cuantitativos, empíricos o racionalistas de la ciencia política y que han mostrado un impresionante desarrollo en Estados Unidos en fechas recientes consideran que la verdadera ciencia 
política apenas está naciendo y que todavía nos encontramos en la prehistoria de la disciplina.22 Con esta posición, parece cancelada de antemano la comunicación entre los diferentes sectores de la ciencia política.

Lo paradójico es que por esta última vía, lo que la ciencia política gana en rigurosidad lo pierde en autonomía, pues es de sobra conocido que el tratamiento de lo político operado por la mayoría de estos sectores científicamente "duros" parte primordialmente de la incorporación de métodos y asunciones provenientes de la economía; es decir, de aquella disciplina social que en términos científicos ha conocido los avances más consistentes. No por casualidad, muchos de los investigadores identificados con esta corriente gustan de ser ubicados bajo el rubro de "análisis económico de la política".

¿Cómo está cambiando entonces la concepción dominante de la ciencia política? Personalmente, considero que no debe echarse en saco roto la exhortación de Almond y de otros politólogos identificados primordialmente con la primera etapa de la ciencia política empírica. Así, no obstante que un núcleo importante dentro de la disciplina se aleja de sus antecesores para caminar hacia el perfeccionamiento de metodologías y de técnicas de investigación cuantitativa altamente complejas, la única vía que permite avanzar hacia una nueva ciencia política para un nuevo mundo, es decir, una ciencia política capaz de ofrecer explicaciones coherentes de los actuales fenómenos globales inéditos, es el de la complementariedad interdisciplinaria y la comunicación y el pluralismo teórico intradisciplinario. Éstas son, pues, las dos posiciones en disputa. Huelga decir que el futuro de la ciencia política depende mucho de la concepción de la disciplina que alcance el mayor consenso en el corto plazo.

\section{Notas}

1. N. Bobbio, "Scienza Politica", en N. Bobbio, N. Matteucci, G. Pasquino (eds.), Dizionario di Politica, Turín, UTET, 1983, PP. 1025-1026.

2. Cfr. A. Ryan, "Socialism for the Nineties", Dissent, Nueva York, vol. 37, núm. 4, 1990, pp. 436-443; K. Tester, "The Collapse of Existing Socialism", Telos, Nueva York, núm. 83, 1990, pp. 151-161: D. Nelson, "The Soviet Union and Europe", Telos, Nueva York, núm. 84, 1990, pp. 124-154.

3. En otro trabajo me he ocupado de las transformaciones que es posible advertir en América Latina como producto de las actuales condiciones mundiales. Véase C. Cansino y V. Alarcón Olguín, América Latina: ¿Renacimiento o decadencia?, San José, FLACSO-Costa Rica/ CIDE, 1994.

4. N. Bobbio, op. cit., p. 1021.

5. De acuerdo con Luigi Graziano, la ciencia política es un campo de estudio que ha encontrado una institucionalización más o menos completa en la 
división del trabajo académico según recorridos temporales y diversos de un país a otro. Las dimensiones que para este autor definen la evolución de la ciencia política son el desarrollo teórico y la institucionalización académica. L. Graziano, Introducción a L. Graziano, D. Easton y J. Gunnell (eds.), Fra scienza e professione. Saggi sullo sviluppo della scienza politica, Milán, Franco Angeli, 1991, p. 8.

6. Para el caso de Estados Unidos véanse D. Easton, "Political Science in the United States. Past and Present", International Political Science Review, vol. 6, núm. 1, 1985, pp. 133-152; A.W. Finifter (ed.), Political Science: The State of the Discipline, Washington, American Political Science Association, 1983; G. Almond, A Discipline Divided. Schools and Sects in Political Science, Newbury Park, Cal., SAGE, 1990. Para el caso de Canadá véanse J.E. Trent, "Factors Influencing the Development of Political Science in Canada: A Case and a Model", International Political Science Review, vol. 8, núm. 1, 1987, pp. 9-24. Para el caso de Europa Occidental véase R. Rose, "Institutionalizing Professional Political Science in Europe. A Dynamic Model", European Journal of Political Science, núm. 18, 1990, pp. 581-603; D. McKay, "Why is there a European Political Science?", ECPR News, marzo de 1990, pp. 29-31. Para mayor información por países véanse L. Morlino, "Political Science in Italy: Tradition and Empiricism", European Journal of Political Research, núm. 20, 1991, pp. 241-258; E.C. Page, "British Political Science and Comparative Politics", Political Studies, vol. 38, 1990, pp. 438-452; J. Leca, "La scienza politica in Francia. Alcune riflessioni sull'organizzazione intellettuale della disciplina in rapporto al suo contesto storico e sociale", en L. Graziano, D. Easton y J. Gunnell (eds.), op. cit., pp. 17-62; H. Kastendiek, "Political Development and Political Science in West Germany", International Political Science Review, vol. 8, núm. 1, 1987, pp. 25-40; J.M. Vallés, "La scienza politica nella Spagna contemporanea: una veduta d'insieme", en L. Graziano, D. Easton y J. Gunnell (eds.), op cit., pp. 143-169; D. Anckar, "Political Science in the Nordic Countries", International Political Science Review, vol. 8, núm. 1, 1987, pp. 73-84. En lo que respecta a América Latina véanse M.C. Guiñazú y M.A. Gutiérrez, "Political Science in Argentina: from Instability to Transition", Bulletin, The Canadian Political Science Association, vol. 20, núm. 2, Laval University, Quebec, mayo de 1991; C. Cansino, R. Maggi, H. Zamitiz (comps.), La ciencia política en México. Estado actual y perspectivas, México, Facultad de Ciencias Políticas y Sociales, UNAM, 1986; B. Lamounier (ed.), A Ciência Politica nos Anos 80, Brasilia, Universidade de Brasilia, 1982. En lo que se refiere a Europa del Este véase J. Tarkowski, "Sienza politica e sociologia: due diverse risposte alla crisi polaca (1980-1987)", en L. Graziano, D. Easton y J. Gunnell (eds.), op. cit., pp. 231-264. Para el caso de África véase A. Jinadu, "La scienza politica nell'Africa anglofona: contesto e logica di sviluppo in prospettiva storica", en L. Graziano, D. Easton y J. Gunnell (eds.), op. cit., pp. 301-329.

7. C. Pateman, "A New Democratic Theory? Political Science. The Public and the Private", ponencia presentada en el XV IPSA World Congress, Buenos Aires, 21-25 de julio de 1991.

8. En un texto reciente, Lawrence Mayer advertía algunas de las implicaciones 
negativas de haber abandonado los estudios comparados en favor de los estudios de aspectos cada vez más específicos. De igual modo, son sugerentes sus observaciones sobre cómo es posible y por qué es deseable superar las contradicciones y límites característicos de este sector de investigación dentro de la ciencia política. L.C. Mayer, Redefining Comparative Politics. Promise versus Performance, Newbury Park, Cal., SAGE, 1989. Sobre este punto véanse también G. Sartori, "Dove va la Scienza Politica?", en L. Graziano (ed.), La Scienza Politica in Italia. Bilancio e prospettive, Milán, Franco Angeli, 1984, pp. 98-114; J. Lane y S. Ersson, "Comparative Politics: From Political Sociology to Comparative Public Policy", en A. Leftwich (ed.), New Developments in Political Science. An International Review of Achievements and Prospects, Aldershot-Vermont, Edward Elgar \& Gowe, 1990, pp. 61-81.

9. T.S. Kuhn, The Structure of Scientific Revolutions, Chicago, University of Chicago Press, 1962. Véase también R. Farfán, "La repercusión de los conceptos de 'paradigma' y 'ciencia normal' de Thomas $\mathrm{S}$. Kuhn en las ciencias sociales”, Sociológica, México, UAM-Azcapotzalco, vol. 3, núms. 7/8, mayo-diciembre de 1988, pp. 45-86.

10. Cf. L.C. Mayer, op. cit., pp. 291-292. Véase también R. Inglehart, "Changing Paradigms in Comparative Political Behavior", en A.W. Finifter (ed.), op. cit., pp. 429-469; W.J. Bluhm, "Introduction: A Call for Paradigm Synthesis", en W.J. Bluhm (ed.), The Paradigm Problem in Political Science. Perspectives form Philosophy and from Practice, Durkhaim, North Carolina, Carolina Academic Press, 1982.

11. D. Easton, op. cit., pp. 140-145.

12. Véase A. Leftwich, "Politics and Development Studies", en A. Leftwich (ed.), op. cit., p. 82.

13. G. Almond, "The Nature of Contemporary Political Science", PS. Political Science and Politics, vol. 23, núm. 1, marzo de 1990, pp. 34-35.

14. Sobre el problema de las mediaciones entre ciencia y poder véanse: L.W. Pye, "Political Science and the Crisis of Authoritarianism", American Political Science Review, vol. 84, núm. 1, marzo de 1990, pp. 3-19; J. Gunnell y D. Easton, "Lo studio dello sviluppo della scienza politica: risultanze e direzioni di ricerca", en L. Graziano, D. Easton y J. Gunnell (eds.), op. cit., pp. 337-338.

15. J. Gunnell y D. Easton, op. cit., p. 335.

16. D. Easton, op. cit., p. 133-152.

17. Entre los principales politólogos que en su momento advirtieron los límites de la ciencia política empírica pueden señalarse G. Almond, "Clods, Clocks, and the Study of Politics", en G. Almond, A Discipline..., op. cit., pp. 32-65 (la primera publicación de este ensayo data de 1977); C.E. Lindblom, "Still Muddling, not yet Trough", Public Administration Review, noviembre-diciembre de 1979; D. Easton, op. cit. Un recuento de los principales cuestionamientos al programa original de la ciencia política empírica puede encontrarse en D. Zolo, La democrazia difficile, Roma, Editori Riuniti, 1989, pp. 46-68. Una crítica más reciente puede encontrarse en P.G. Cerny, The Changing Architecture of Politics, Londres, SAGE, 1990. Según este autor, el estudio sistemático de la política sufre de una profunda ambigüedad y esquizofrenia: la conceptualización teórica de cómo trabajan las institucio- 
nes políticas y de su efecto está muy subdesarrollado. De acuerdo con ello, nos encontramos en una pobre situación para entender las estructuras y evaluar los recientes cambios estructurales en política, economía y sociedad que serán relevantes en el siglo XXI.

18. Nos referimos al libro de D.M. Ricci, The Tragedy of Political Science, New Haven, Yale University Press, 1985. De acuerdo con esta interpretación, la ciencia política en Estados Unidos parece incapaz de producir un "conocimiento político" efectivo precisamente por su empeño en alcanzar un conocimiento cierto y absolutamente preciso --"científico"-- de la vida política. Este hecho desvía simultáneamente al politólogo de los temas cruciales de la sociedad en la que vive, como la crisis de las sociedades democráticas, pues estos temas no pueden ser enfrentados seriamente por quien hace de la neutralidad política su propio hábito profesional.

19. Véase, por ejemplo, D. Zolo, op. cit., Pp. 61-68.

20. N. Bobbio, "Per un mappa della filosofia politica", en D. Fiorot (ed.), La filosofia politica oggi, Turín, G. Giappichelli Editori, 1990, pp. 5-23.

21. G. Almond, $A$ discipline Divided..., op. cit., PP. 13-31. Véanse también H. Eckstein, "A Comment on Positive Theory", PS. Political Science and Politics, vol. 22, núm. 1, marzo de 1989, p. 77; M.T. Gibbons, "Political Science, Disciplinary History and the Theoretical Pluralism: A Response to Almond and Eckstein", PS. Political Science and Politics, vol. 23, núm. 1, marzo de 1990, pp. 44-46; J. Gunnell, "Political Theory. The Evolution of a Sub-Field", en A.W. Finifter (ed.), op. cit., pp. 3-45.

22. Con esta idea surgieron trabajos tan importantes como: W. Riker y P. Ordeshook, An Introduction to Positive Political Theory, Engelwood Cliffs, N.J., Prentice Hall, 1973; J. Buchanan, The Economics of Politics, West Sussex, Institute of Economic Affairs, 1978; J. Ferejohn, B. Cain y M. Fiorina, The Personal Vote, Cambridge, Harvard University Press, 1987. Dos análisis muy ilustrativos del conjunto de presupuestos de este sector de la ciencia política pueden encontrarse en: T. Moe, "On the Scientific Status of Rational Choice Theory", American Journal of Political Science, vol. 23, núm. 1, 1979; G. Almond, "Rational Choice Theory and the Social Science", en G. Almond, A Discipline Divided..., op. cit., pp. 117-136. 